Presidential Address 2020

\title{
REACH AND COUNT EVERY PREGNANT MOTHER AND NEWBORN
}

Kaushalya Kasturiaratchi ${ }^{1}$

The term "Perinatal" refers to the period immediately before and after birth. The perinatal period is defined in diverse ways. According to many definitions, it starts at $20^{\text {th }}$ to $28^{\text {th }}$ weeks of gestation and ends 1-4 weeks after delivery. Perinatal health refers to the health of both the mother and the newborn during this specific period of time. The Sustainable Development Goals (SDGs) were set in 2015 by the United Nations General Assembly, and intended to be achieved by the year 2030 . As you are aware, there are 17 SDGs and the goal applicable for health is the goal 3 i.e. "ensure healthy lives and promote wellbeing for all, at all ages". This goal includes nine major targets and four sub-targets, and 14 indicators. Out of this gamut of targets, two indicators are relevant to maternal and newborn health. Those are the Maternal Mortality Ratio (MMR) and the Neonatal Mortality rate (NMR). 
MMR in Sri Lanka has improved markedly over last several decades. However, it is stagnant over last 10 years around 30 deaths per 100,000 live births (LB) [Figure 1]. Therefore, fresh approaches need to be considered in achieving the country's target of $<10$ deaths per 100,000 LBs in 2030 .

Infant Mortality Rate too has significantly reduced over the years. So does the NMR and the still birth rates [Figure 2]. However, all these indicators have now reached a plateau and further reductions need fresh thinking and innovative approaches.

Given that the periods covered under MMR and NNMR are well within the perinatal period, it is obvious that, this period of time in the life of a mother and a newborn, will have a tremendous impact on their quality of lives. Therefore, the numerical figures that reflects their health such as Maternal Mortality Ratio and Neonatal Mortality Rate are not just statistical entities.

\begin{tabular}{|c|c|c|c|c|}
\hline & 2015 & 2020 & 2025 & $\begin{array}{l}2030 \\
(50 G)\end{array}$ \\
\hline MMR & 33.7 & 25 & 25 & $<10$ \\
\hline
\end{tabular}

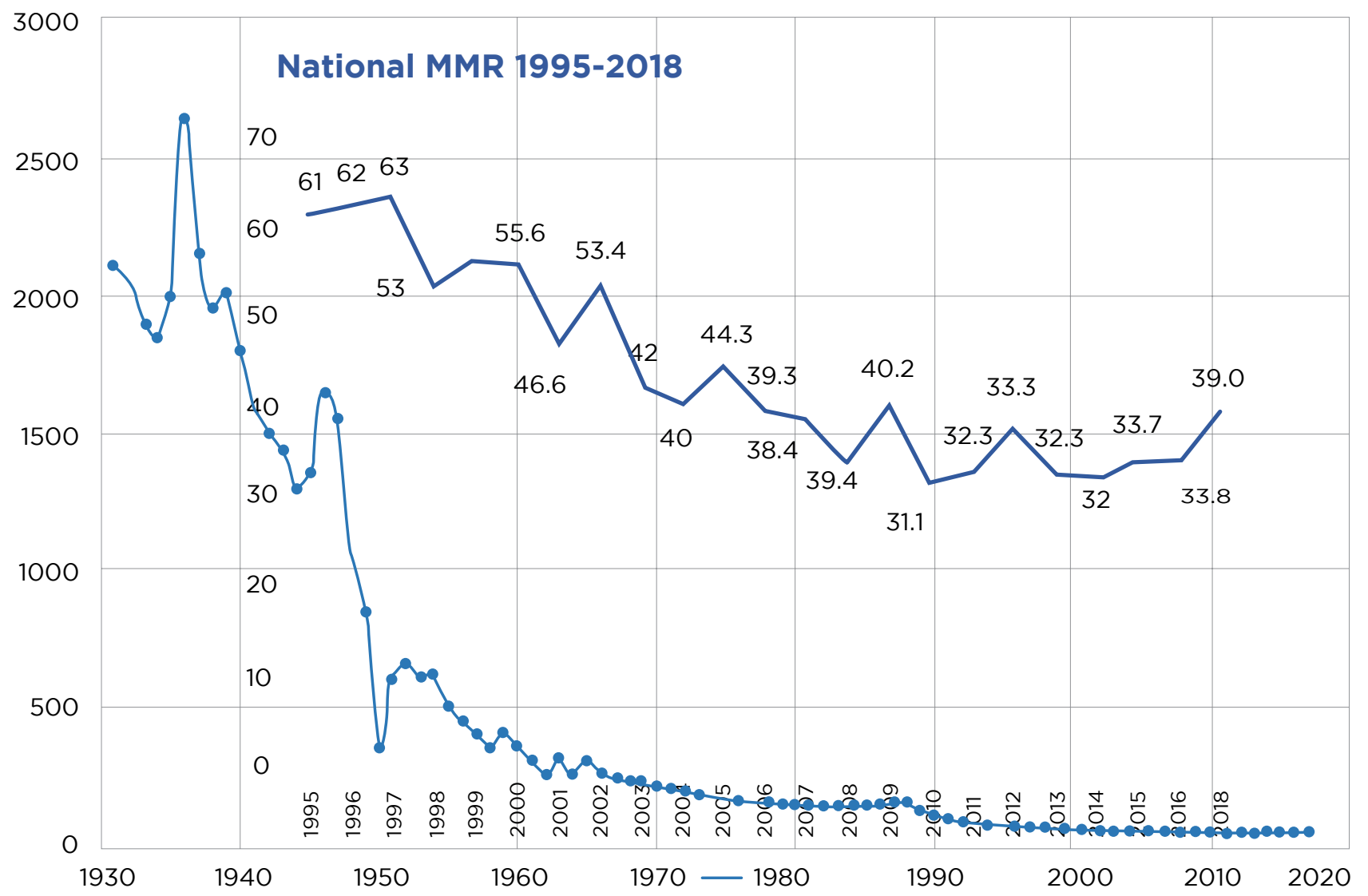

Source : FHB

Figure1: Maternal Mortality Ratio change over the years in Sri Lanka 


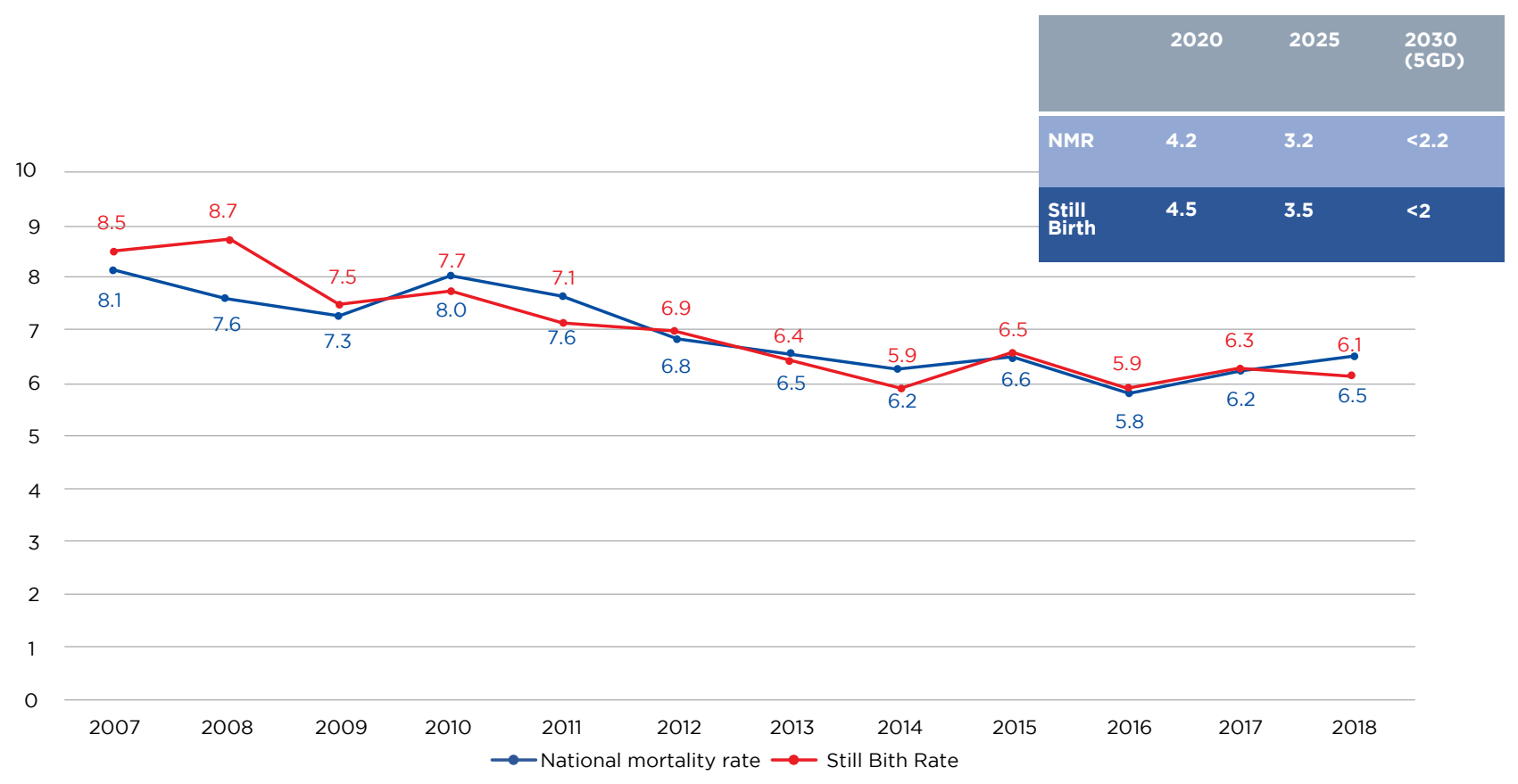

Source : FHB

Figure 2: Infant mortality and stillbirth rates in Sri Lanka

Apart from the influence on these indicators, this period of time, is an emotionally charged period, for the mother and her family. It is an important period for the medical team, as careful monitoring and provision of timely intervention becomes important, to ensure the welfare of both the mother and the newborn. During this period of time, medical teams should pay more focused attention on both mental and physical wellbeing of the mothers and newborns. Appropriate application of interventions, and collection of data by the programme managers is highly important to implement, cost effective evidence based interventions.

Considering this background, the theme introduced for for the year is "Reach and Count every pregnant mother and newborn". Beyond the ordinary meaning of the two terms, reach and count, there is little more technicality associated with these 2 words mentioned. Reaching in this instance means, evidence based interventions, introduction of new technologies and advance therapeutics, which you will realize, is beyond the ordinary reach out for these mothers. Such involves correct decision making, application of technology, and care for both the mother and the newborn. Similarly, counting refers to more intense type of identifying and collecting data with regard to status, progress and health outcomes of these mothers and newborns not necessarily limiting to clinical management but also to facilitate programme efficiency and proper health policy planning. Therefore, the theme for this year, encapsulates, wider multi disciplinary and an action oriented approach.

Having said all these, I would like to introduce what the new council and I as the President, propose that we achieve in the year 2020. Digitalization of perinatal health information, strengthening, perinatal psychiatry and an attempt to lower the cesarean section rates and promotion of normal vaginal deliveries. As these areas involve many 
expertise, we would like to call upon, the members representing the different specialties to join hands with us to plan an execute the related activities. Very briefly, I would now like to elaborate on the three main thematic areas, selected for this year.

\section{DIGITALIZATION OF PERINATAL CARE INFORMATION}

Collection of health information in Sri Lanka has a long history that dates back to 1940s. Medical Statistics Unit of the Ministry of Health was established in mid 1950s and a comprehensive $\mathrm{MCH}$ information system covering the field health services, was started in early 1980s. Even though, we have a long history of collecting health information, the hospital information systems have not been fully developed to the expected levels, where as the Medical Officer of Health based field information systems have evolved tremendously over last 2 decades. More recently, the role of digital health in health system strengthening has been identified, as a valuable contribution, to advance the universal health coverage and other health aims of SDGs. Digital health interventions are expected to complement, and enhance health system functions, through accelerated exchange of information.

Digitalization of Medical Officer of Health based field $\mathrm{MCH}$ information system was initiated in 2017 by the FHB, covering all 6765 Public Health Midwife areas in the country and 4292 field health clinics in 354 Medical Officer of Health areas. Within three months, of implementation of the system, our team managed to achieve $100 \%$ coverage in all Public Health Midwife (PHM) areas and field clinics, enhancing the shift from totally paper based system to a web based electronic system. Each and every, PHM and field clinic data, is now entered into this system monthly. This web based system, was developed and implemented, not utilizing government or any other funds. It is a collective effort of the Health Informatics doctors and the public health doctors attached to the Monitoring \& Evaluation Unit of the Family Health Bureau. For this system, the team won the Common wealth digital awards in 2 consecutive years 2017 and 2018 and was nominated for BMJ awards in 2017. In 2019, we won the eSwabimani digital awards and were nominated for the World Summit Awards.

Following this success story, we extended the electronic Reproductive Health Management Information system (eRHMIS) to hospital settings in mid 2019, to capture information from private hospitals in Sri Lanka. This was done in collaboration with the Perinatal Society of Sri Lanka and Perinatal Association of Private Hospitals in Sri Lanka. Having information from private sector was a long felt need of the country. Until now, we had no idea about the contribution of private hospitals in maternal and newborn health. This has been identified as a major deficiency in many instances, and recently during the application process, for WHO certification on elimination of mother to child transmission of HIV and Syphilis. This system is now running successfully covering all major private hospitals in Colombo and will be expanding further in 2020 to other districts in collaboration with the PSSL and Perinatal Association of Private Hospitals in Sri Lanka. 
In addition, development and implementation of Neonatal ICU information system including an information system for tracking therapeutic cooling and nitric oxide therapies will be undertaken in the Level 3 and $3+$ healthcare institutions during this year.

\section{STRENGTHENING PERINATAL PSYCHIATRY}

There is no time in the lifespan, that the statement "there is no health without mental health" rings truer than in the perinatal period. Pregnancy, and the arrival of a new baby is a challenging time for mothers. This period can be over shadowed by the appearance of mental illnesses associated with widespread stigma. Currently, not much attention has been paid to this aspect of mother's health. There are many psychological issues identified in this period and they range from baby blues to perinatal depression to suicides. Available literature indicates that in Low and Middle Income Countries, an estimated 15-20\% of females' experience perinatal depression. Magnitude of the problem, varies from country to country and in a study done in Sri Lanka, it was shown to be $16.2 \%$ which is a considerable health burden. Mothers with perinatal depression are less likely to take care of themselves have threatened relationships with their partners and demonstrate impaired ability to work. They are less likely, to seek and receive antenatal care and also has a risk of committing suicides.

Perinatal mental disorders are associated with, increased risk of psychological and developmental disturbances in children. These mothers with psychiatric disorders, will find it difficult to bring up children, and there by causing physical, cognitive, social, behavioural and emotional developmental problems for their children. Higher rates of Low Birth Weights, malnutrition, stunting and other diseases have also been observed among these children. Negative effects can extend up to adolescent period causing adolescent depression, depressive disorders and poor social competencies. Suicides occupies, the one end of the spectrum of mental health disorders, during perinatal period. Globally, maternal suicides are a leading cause of maternal mortality across all income settings. Available information suggests prevalence of maternal suicides ranges from $0 \%$ in Vietnam to $23.08 \%$ in Argentina with an estimated global average of $1.68 \%$ of pregnancyrelated deaths. In Sri Lanka, around 25 deaths are reported annually, with a rate of 8.3 per 100,000 LB in 2017 [Figure 3]. Currently, we do not take in to consideration, maternal suicides as a direct cause of maternal deaths. However, ICD MM necessitate, the counting suicides during pregnancy and postpartum period. If suicides are also included, there will be a further increase of MMR in SL.

Analysis of Psychological autopsies of 409 maternal suicides from 2002 to 2017 , have shown that More than $50 \%$ the suicides have occurred in antenatal period, majority in the first pregnancy, and $75 \%$ of mothers were below 30 years of age.

Currently, mothers are being screened for postpartum depression in field setting, however, the detection rates are very low. 
What we intend to do regarding perinatal mental health during 2020 includes, integrating perinatal psychiatry services into the maternal care package, introduce mental health education sessions during antenatal period, enhancing self identification of depression and help seeking, community based interventions, using a reliable tool to identify mental health problems during pregnancy and following birth of the child, and training of healthcare workers to identify symptoms and signs of mental health problems, providing counselling about stress and providing effective psychological support with community engagement, enhancing family support is challenging unless paternity leave is provided to husbands for their active involvement during perinatal period.

\section{LOWERING THE RISING TRENDS IN CESAREAN SECTION RATES}

Caesarean sections (CS) are the most common surgery worldwide, and it is a procedure that can save lives of women's and babies', when complications occur during pregnancy or child birth. The use of CS, for non-medically indicated reasons is a cause for concern because, the procedure is associated with considerable short-term and long-term adverse effects and rising health-care costs. Risks are progressively increasing as the number of previous caesarean deliveries, increases. Based on systematic reviews, WHO has concluded that $\mathrm{CS}$ rates of greater than $10 \%$ are not linked with lowering maternal and neonatal mortality rates. CS use is growing at an "alarming" rate globally, accounting for $21 \%$ of births being CS in 2015 .
A series of papers published in the Lancet shows that CS rates above $10 \%$ to $15 \%$, will not contribute for better health outcomes rather it indicates poor quality of service provision.

CS rates exceeds $40 \%$ in only 15 countries in the world and highest rate of $58.1 \%$ has been reported in Dominican Republic. In Sri Lanka, CS rates are increasing alarmingly and reaching the highest rates reported globally. In $2019,>50 \%$ of CS rates are reported in 40 Medical Officer of Health areas. Highest being the Kurunegala Municipal Council with a rate of $67 \%$ in 2019 . Nearly 40 hospitals in Sri Lanka, have reported, CS rates over $40 \%$ in 2018 . Can we afford to have such increase is questionable? Efforts should be taken, to strengthen the clinical decision making, based on empirical evidence and following measures have been suggested by WHO to reduce high rates of Cesarean Sections. Application of Robson classification and assess the underline causes at the institution level, setting institutional targets, based on data and development of plan, for institution based action. Non clinical interventions, targeted at pregnant women, healthcare professionals, and community, aiming at reducing CSs. In addition to the activities, under the three themes, the following, have also being listed for 2020 .

National Capacity building Programmes \& Workshops specially aiming at building capacity of PHMs and Nurses. Capacity building on Positive experience in pregnancy, Respectful labour care, Pain control in labor, NICU Care and Breast feeding and completion of some of the last year's activities, developing a training module, and training programmes on therapeutic cooling 


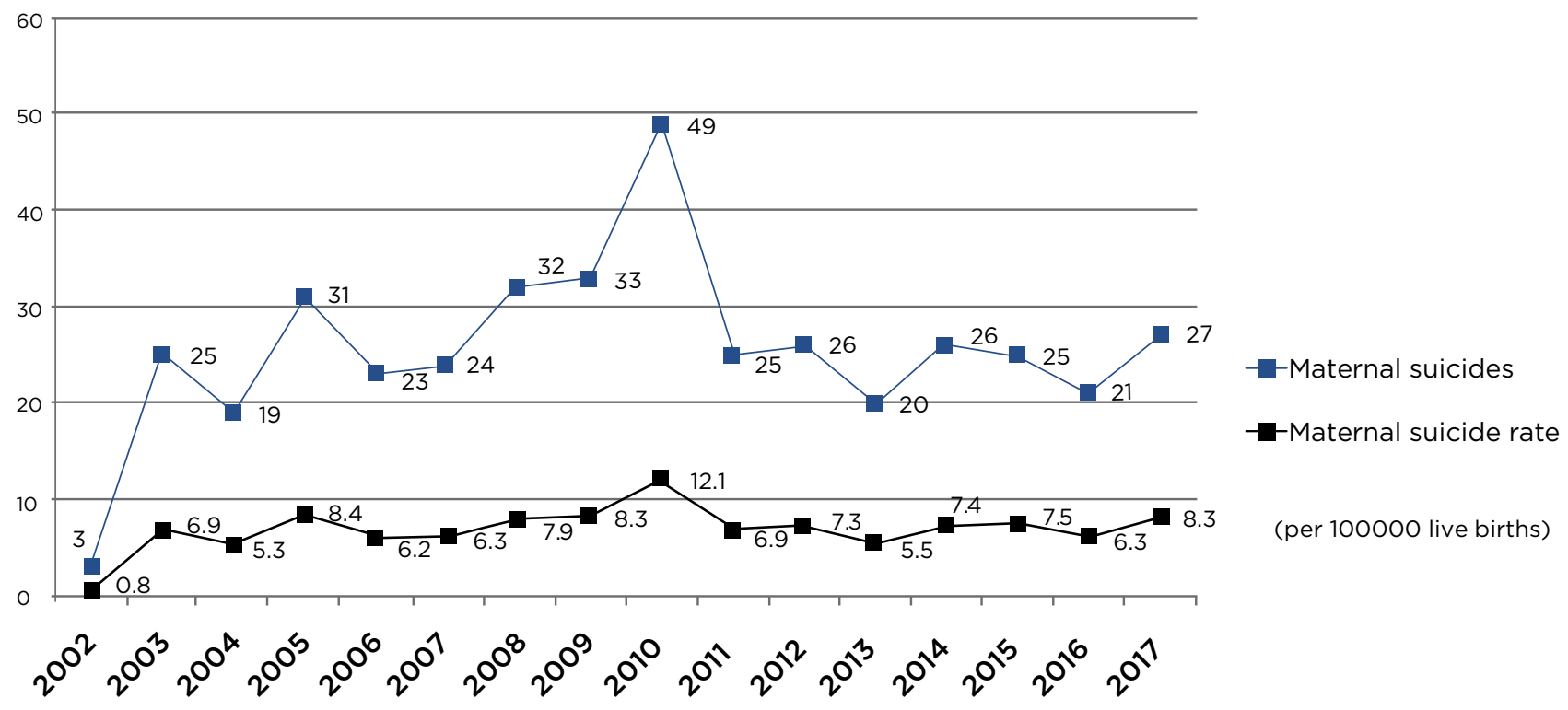

Source : FHB

Figure 3: Numbers and rates of maternal suicides from 2002- 2017

and nitric oxide therapies, NICU module, and neonatal formulary initiated under Dr. Surantha Perera's Presidency.

PSSL will be working in collaboration with the Perinatal Association of Private Hospitals in Sri Lanka, Nurses Forum and the PHMs Forums during the year.

Additionally, PSSL will be working in collaboration with all other Academic Colleges to accomplish the activities under the three themes. Annual Scientific Sessions will be held from 11th to 12 th November 2020 at Hotel Galadari.

To recapitulate, the theme for the next year is "Reach and count every mother and newborn, and we look at, the different aspects, that come under the two terms, "Reach and count". As mentioned before, these are tasks, can be implemented, by multi disciplinary teams, and I feel that the expertise available with the PSSL is optimal in developing these approaches.

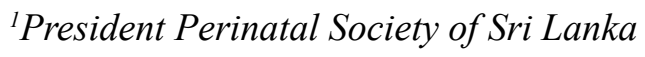

Consultant Community Physician

Family Health Bureau, Ministry of Health

Sri Lanka 